NASA Technical Memorandum 103133

\title{
Lateral Spreading of Au Contacts on InP
}

Navid S. Fatemi

Sverdrup Technology, inc.

Lewis Research Center Group

Brook Park, Ohio

and

Victor G. Weizer

National Aeronautics and Space Administration

Lewis Research Center

Cleveland, Ohio

Prepared for the

1990 Spring Meeting of the Materials Research Society

San Francisco, California, April 16-21, 1990

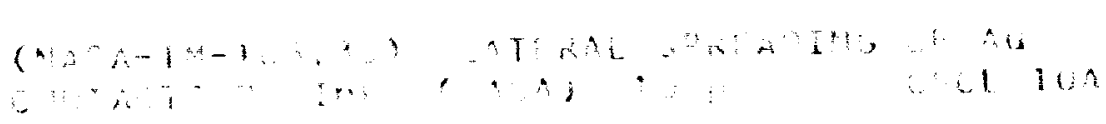

$0,1+4$ uncl on. 
LATERAL SPREADING OF AU CONTACTS ON InP

\author{
Navid S. Fatemi* \\ Sverdrup Technology, Inc. \\ Lewis Research Center Group \\ Brook Park, Ohio 44142 \\ and \\ Victor G. Weizer \\ Nationá1 Aeronautics and Space Administration \\ Lewis Research Center \\ Cleveland, Ohio 44135
}

\title{
ABSTRACT
}

We have investigated the contact spreading phencmenon observed when small area Au contacts on InP are annealed at temperatures above about $400 \mathrm{c}^{\circ}$. We have found that the rapid lateral expansion of the contact metallization which consumes large quantities of InP during growth is closely related to the third stage in the series of solid state reactions that occur between InP and Au, i.e., to the $A_{3}$ In-to-AugIn $_{4}$ transition. We present detailed descriptions of both the spreading process and the $\mathrm{Au}_{3}$ In-to-AugIn 4 transition along with arguments that the two processes are manifestations of the same basic phenomenon.

\section{INTRODUCTION}

When small area Au-based contacts on InP are sintered at temperatures above $400{ }^{\circ}$, a rapid lateral spreading of the metallization along specific crystallographic directions on the semiconductor surface can be observed. $[1,2]$ The material in the spread regions has been identified as the compound AugIn $4,[1]$ and the extent to which the spreading occurs has bien shown to be related to the amount of Au originally present. [2] The process is apparently thermally activated with an activation energy samewhere between 5.6 and $11.6 \mathrm{eV}$.[2] The purpose of this paper is to provide a more detailed description of the spreading phenomenon in the Au-InP system and to show that it is closely related to the pink-to-silver $\left(\mathrm{Au}_{3} \mathrm{In}\right.$-to-Aug $\left.\mathrm{In}_{4}\right)$ transition that is observed to take place during contact sintering.

\section{CONTACT SPREADING}

The spreading phenamenon is illustrated in figure 1 where we show the results of heating a $2000 \&$ thick, 30 micrometer diameter circular gold pad deposited on a polished, (100) oriented InP surface for 2.5 hours at $435{ }^{\circ}$. As cin be seen, the metal-semiconductor contact area has been increased by more than a factor of two. It can also be seen that a significant amount of InP surrounding the circular pad has been replaced by the silver-colored metallization. The metallization, which has been identified as AugIn 4 by Keramidas, et al.[1], can be seen to be more or less flush with the unreacted InP along the reaction front. Closer to the original disc, however, the surface of the metallization drops considerably below the level of the surrounding InP. Since the 
disc itself is not observed to increase in size, the drop in the level of the metal indicates that spreading is accampanied by a significant mass loss.

The amount of InP dissolved in the reaction can be determined by chemically removing the metallization in the spread regions. The resulting topography (for an identically processed sample) is shown in figure 2. What we find when we remove the metallization is that the InP has been eroded to an undulating, but on the average constant, depth. The metallization in the spread region is therefore much thicker near the reaction front than it is closer to the disc. Fram the orientation of pits that were etched in the InP surface with a bromine-methanol etchant we have determined that the (111) plane defining the reaction front of the spread region is the In-faced plane. More importantly, the spread regions were found to contain no trace of the $\mathrm{Au}_{2} \mathrm{P}_{3}$ layer that has been observed to form at the metal-semiconductor interface during contact sintering. $[3,4]$

With regard to the growth dymamics, we found that the spreading process requires an incubation time at temperature before growth begins. The incubation time appears to be material sensitive, low dislocation density material being more susceptible to spreading than lower quality material.

As we followed the progress of spreading we noted that the spreading regions were always silver in color, and that when spreading starts at a given point on the periphery of a disc, the adjacent regions of the disc are also silver in color. As spreading proceeds, the entire disc changes fram pink to silver.

It is evident that during the spreading process Au is being transported from the Au-rich disc to the reaction front in the spread region, a considerable distance away. However, if the only mass flow during spreading were the diffusion of Au from the disc to the reaction front, then at the front

$$
9 \mathrm{Au}_{i}+4 \mathrm{InP} \rightarrow \mathrm{AugIn}_{4}+4 \mathrm{P} \text {. }
$$

This reaction would result in a $28 \%$ increase in the volume of the spread regions, even if the $P$ atams dissipated. Since we actually see a substantial decrease in the volume of the metal in the spread regions, we must conclude that In is being transported from the reaction front to the disc.

We can get an estimate of the spreading rate through the relationship $I=(D t)^{1 / 2}$. Here $D$ is the diffusion coefficient, $t$ is the diffusion time, and $\mathrm{L}$ is the distance between the disc and the reaction front (which averages about 10 micrameters for samples such as those in figures 1 and 2). Considering a growth time of 1.5 hours at $435{ }^{\circ} \mathrm{C}$, we calculate a diffusion coefficient of $2 \times 10^{-10} \mathrm{~cm}^{2} / \mathrm{sec}$. This value is in basic agreement with spreading rate measurements made by Elias, et al.[2]

When we sought to determine the effect of an $\mathrm{SiO}_{2}$ cap layer on the spreading process, we found that placing an $\mathrm{SiO}_{2}$ on the disc itself did not suppress spreading. However, while the cap layer does not affect the ability of a disc to spread when it is deposited on the disc, it is very effective in preventing spreading when deposited on the InP surface adjacent to the disc. Figure 3, for instance, shows the spreading that emanated fram three partially capped $\left(600 \AA \mathrm{SiO}_{2}\right)$ Au discs (2000 \& thick) after 1 hour at $442{ }^{\circ} \mathrm{C}$ in forming gas. As can be seen, spreading took place only into uncapped InP. Figure 4 demonstrates how spreading fram 
an uncapped disc is terminated upon encountering an $\mathrm{SiO}_{2}$ cap on the InP surface.

While a cap layrer is effective in stopping the advance of spreading, it does not do so abruptly. A close look at the intersection of the spread region with capped InP reveals that the spread region tunnels under the cap for about 1 micrometer before stopping. An interesting feature of this tunnelling is that the metallization under the cap does not shrink in volume as does the uncapped metallization. Figure 5 illustrates the planar character of the metallization under the cap (removed for clarity) as campared to that along the adjacent uncapped reaction front. While the details of the reaction suppressing mechanism are not completely clear, it is apparently associated with the cap's effect on the escape of phosphorus.

\section{THE AU-InP INIERACTICN PROCESS}

As stated in the introduction, there is evidence that the spreading phenomenon is closely related to the third stage in the Au-InP interaction. Let us first review what is known about the Au-InP reaction mechanisms and then draw same comparisons.

Recent investigations have shown that the solid state reaction of Au with InP takes place in several distinct steps or stages. $[3,4]$ The first two stages have been shown to involve the entry of In and $P$ into the contacting Au metallization. In stage I, In enters the Au lattice via a dissociative diffusion mechanism, the rate of which is controlled by the vacancy generation rate at the free surface of the metallization. In entry continues until the amount of In in the Au lattice reaches the solid solubility limit (about 108). Stage I is quite rapid. Its results can be observed after aging at room temperature for several months. The $P$ atcms that enter the metal with the In either take non-lattice sites in the metal or exit the system.

If stage $I$ is allowed to go to completion a second In entry mechanism becomes active. [4] This stage proceeds rapidly in the temperature range usel for contact sintering. In this stage In continues to enter the metalliziation, but this time via an interstitial exchange or kickout mechanism. In entry continues until the metallization is converted from the $10 \mathrm{z} \mathrm{Au}(\mathrm{In})$ saturated solid solution to the compound $\mathrm{Au}_{3}$ In. The rate limiting process in stage II has been shown to be the release of In fram the InP and its insertion interstitially into the Au lattice. Whereas in stage I the $\mathrm{P}$ atams released with the In leave the system without reacting, in stage II they react to form the compound $\mathrm{Au}_{2} \mathrm{P}_{3}$ at the metal-seniconductor interface.

We report here on the results of an investigation into a third stage in the Au-InP reaction that takes place upon further sintering. In this stage In continues to enter the Au lattice with the result that the pink compound $\mathrm{Au}_{3} \mathrm{In}$ is converted to a silver colored compound. Since there are a number of stable Au-In compounds with higher In content than $\mathrm{Au}_{3}$ In [5], our first task was to identify the silver colored product of stage III. The results of an x-ray photoelectron spectroscopy (XPS) analysis indicated that the In content of the silver alloy is close to 30 atz. The endpoint of stage III was thus identified as AugIn 4 .

our next task was to investigate the kinetics of the stage III reaction. In order to study the events occurring during this stage without interference from stages I and II, we deposited Au In (the stage 
II product) on a number of InP substrates and observed the behavior of this system at elevated temperatures. When we measured the rate at which the pink $\mathrm{Au}_{3}$ In was converted to the silver AugIn 4 at a given temperature, we found that it (the conversion rate) was proportional to the area of the interface between the two phases. Thus if the measured pink-tosilver conversion rate $\left(\mathrm{cm}^{3} / \mathrm{sec}\right)$ is divided by the corresponding interphase area, the result is a conversion rate per unit area $(\mathrm{cm} / \mathrm{sec})$ that is invariant with sintering time. A plot of the logarithm of this rate versus the reciprocal temperature (figure 6) yields an activation energy of $11.3 \mathrm{eV}$.

We have found that there is a major difference in the behavior of the phosphorus atams released into the metallization during stage III as compared to stage II. In Au-contacted and annealed samples where stage II has gone to completion, one abserves the presence of the campound $\mathrm{Au}_{2} \mathrm{P}_{3}$ at the InP-metal interface when the $\mathrm{Au}$-In alloys are chemically removed. [3] However, after the above experiments where stage II is bypassed by depositing and annealing $\mathrm{Au}_{3} \mathrm{In}$, no sign of $\mathrm{Au}_{2} \mathrm{P}_{3}$ is found. Thus in stage III (as in stage I) the $P$ atoms released into the contact metal either take non-lattice sites, or they leave the system.

\section{THE EQUTVALENCE OF STAGE III AND CONTACT SPREADING}

From the preceding analysis we find that stage III and contact spreading have a great deal in cammon. 1) In both cases the reaction product is $\mathrm{AugIn}_{4}$. 2) The activation energies for the two processes agree within published limits of uncertainty. 3) In neither case does the interfacial $\mathrm{Au}_{2} \mathrm{P}_{3}$ layer form. 4) Stage III conversion in the deposited Au disc is observed to take place concurrently with the nucleation and growth of the spread regions in the adjacent InP.

On the basis of these similarities, it seems reasonable to conclude that spreading and the stage III reaction are manifestations of the same phase transformation process.

\section{THE SPREADING/STAGE III MECHANISM}

We have shown, in the preceding analysis, that the spreading mechanism involves the transport of both $\mathrm{Au}$ and In in opposite directions through the metallization in the spread region. It is well known that In diffuses interstitially in all the intermediate Au-In alloys. [6] As in stages I and II reactions, the In diffusion mode in stage III is therefore also interstitial. In addition, since Au diffuses interstitially in pure In [7] and also in the closely related sn [8] and $\mathrm{Au}-\mathrm{Sn}$ alloys $[9,10]$, it is reasonable to assume that the Au diffusion mode in Au-In alloys is also interstitial.

The above arguments suggest that both the diffusion of $\mathrm{Au}$ to and In fram the spread front is interstitial. Such interstitial interdiffusion in metals is a characteristic of an interstitial exchange or kickout mechanism. [11]

Let us recall the characteristics of the kickout process. This process, which has already been proposed to explain the stage II phase transformation, consists of a number of steps. [4] It starts with the release of In from the InP and its entry into the metallization as an interstitial. After entry the In interstitial diffuses rapidly to the 
phase boundary where energy considerations favor an interstitialsubstitutional interchange. In this interchange, called the kickout step, the In interstitial displaces a Au substitutional atom and takes its place on the metal lattice. The resulting $\mathrm{Au}$ interstitial then diffuses away from the kickout site to react (in the case of stage II)with unbound $\mathrm{P}$ atcms to form $\mathrm{Au}_{2} \mathrm{P}_{3}$. The unique characteristic of this process is the fact that both species ( $\mathrm{Au}$ and $\mathrm{In}$ ) diffuse interstitially (and thus very rapidly).

We thus propose that the spreading process consists of the interstitial entry (at the spread region reaction front) and diffusion of In in the metal lattice, and its exchange with a substitutional Au atom (kickout) at the $\mathrm{Au}_{3} \mathrm{In}-\mathrm{Au}_{9} \mathrm{In}_{4}$ interphase boundary in the Au-rich disc where $\mathrm{Au}_{3} I n$ is being transformed to $\mathrm{Au}_{9} I n_{4}$. The reaction at the interphase boundary is proposed to proceed as

$$
13 \mathrm{Au}_{3} \mathrm{In}+3 \mathrm{In}_{\mathrm{i}} \rightarrow 4 \mathrm{Au}_{9} \operatorname{In}_{4}+3 \mathrm{Au}_{\mathrm{i}}
$$

where the subscript indicates interstitial siting. The resulting Au interstitials are transported back to the reaction front where they combine with interstitial In and vacant lattice sites to form AugIn 4 there.

The proposal that a kickout mechanism is active during stage III provides an explanation for the observed dependence of the stage III reaction rate on the area of the interphase boundary. If the kickout rate is proportional to the number of potential kickout sites, and if these sites are locatiod only at the boundary between the two phases, it follows that the kicksut rate should indeed be proportional to the $\mathrm{Au}_{3} \mathrm{In}-\mathrm{Au}_{9} \mathrm{In}_{4}$ interfacilal area.

As mentioned, $P$ atoms released at the reaction front along with In do not react chemically during the spreading process and thus are free to exit the system. If these atams (and/or the vacancies they leave behind at the InP-metal interface) are slow in diffusing out of the system, a slow (but sizeable) decrease in the volume of the metallization would be observed with time. ".mis is, in fact, what is observed. As we have mentioned previously, the newly generated metallization near the reaction front in the spread regions is considerably thicker than the metal remote fram the front where the $P$ atams and/or the vacancies they left behind have had time to diffuse out of the system. It is felt that the effect of InP capping on the geametry of the spreading metallization (figure 5) is related to its ability to affect the ease of phosphorus escape.

\section{REFERENICES}

1. V. G. Keramidas, F. Temkin, S. Mahajan, Inst. Phys. Conf. Ser. $\underline{56}$, 293 (1981)

2. K. R. Elias, S. Mahajan, C. L. Bauer, A. G. Milnes, W.A.Bonner, J. Appl. Phys. 62, 1245 (1987)

3. N. S. Fatemi and V. G. Weizer, J. Appl. Phys. 65, 2111 (1989).

4. N. S. Fatemi and V. G. Weizer, J. Appl. Phys. 67, 1934 (1990).

5. F. A. Shunk, Constitution of Binary Alloys, second supplement, (MOGraw Hill, New York, 1969) p 72.

6. G. W. Powell and J. D. Braun, Trans. AIME 230, 694 (1964).

7. T. R. Anthony and D. Turnbull, Phys. Rev. 151,495 (1966).

8 . B. F. Dyson, J. Appl. Phys. 37, 2375 (1966). 
9. M. Creydt and Fichter, Metall (Berlin) 25, 1124 (1971).

10. L. Buene, Thin Solid Films 47, 159 (1977).

11. W. Frank, U. Gosele, H. Mehrer, and A. Seeger, Diffusion in Crystalline Solids, G. E. Murch and A. S. Nowick, Eds., (Academic Press, Orlando, 1984) p 63.

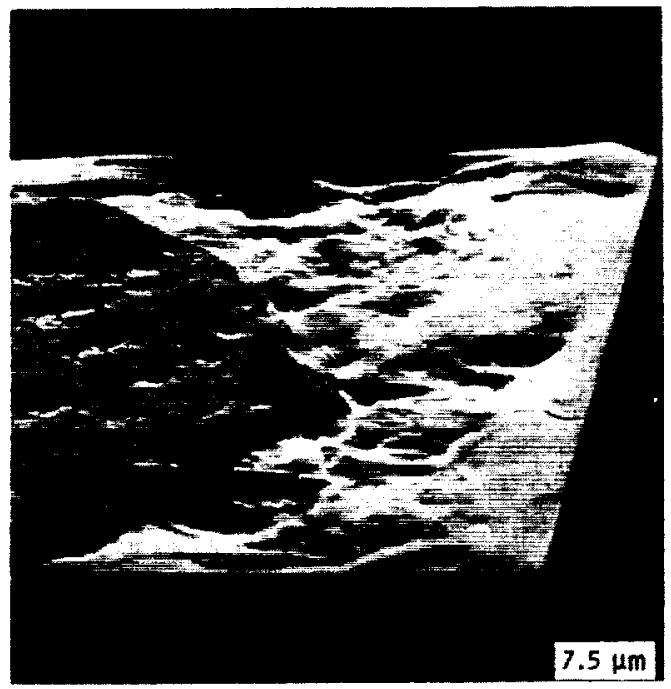

FIGURE 1. - CONTACT SPREADING 1.5 HOURS AT $435{ }^{\circ} \mathrm{C}$

ORIGINAL PAGE

BLACK AND WHITE PHOTOGRAPH

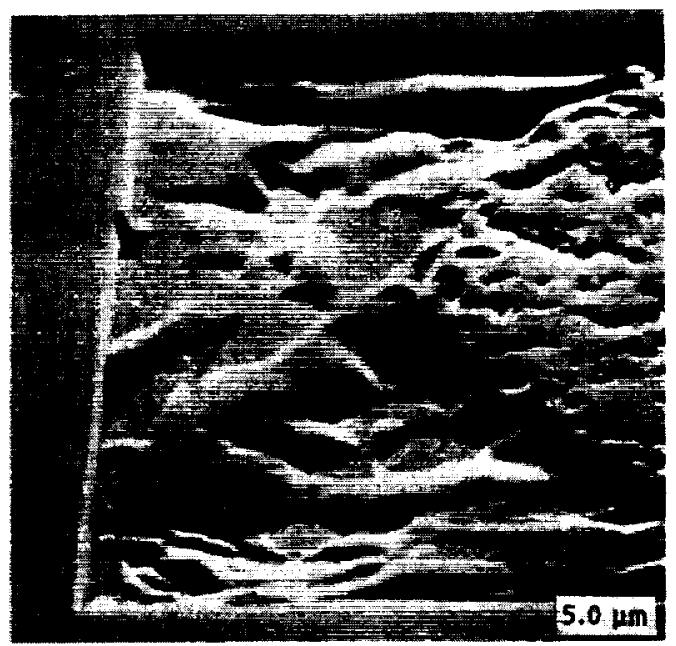

FIGURE 2. - InP TOPOGRAPHY AFTER REMOVAL OF METALLIZATION FROM SPREAD REGION. 


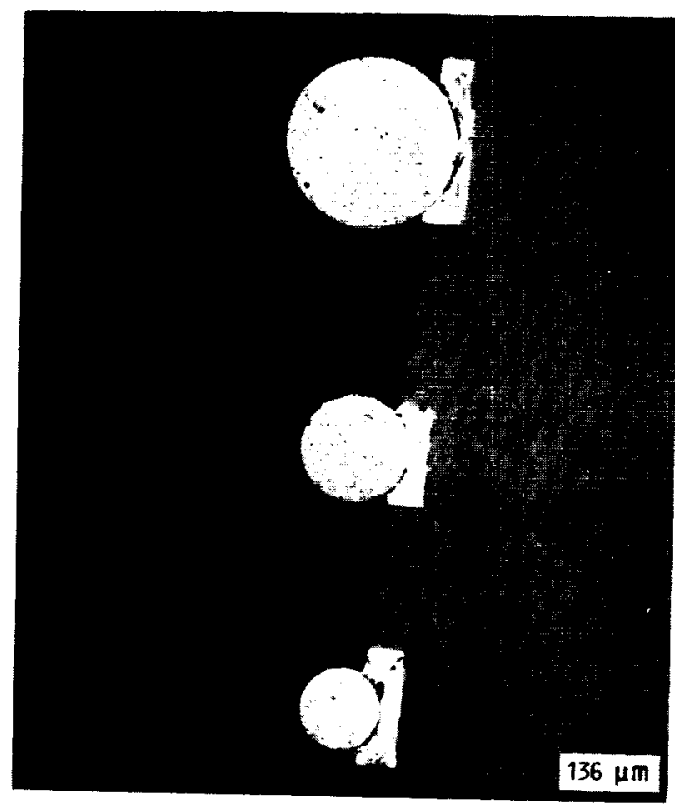

FIGURE 3. - SPREADING FROM PARTIALLY CAPPED CONTACTS.

\section{ORIGINAL PAGE \\ BLACK AND WHITE PHOTOGRAPH}

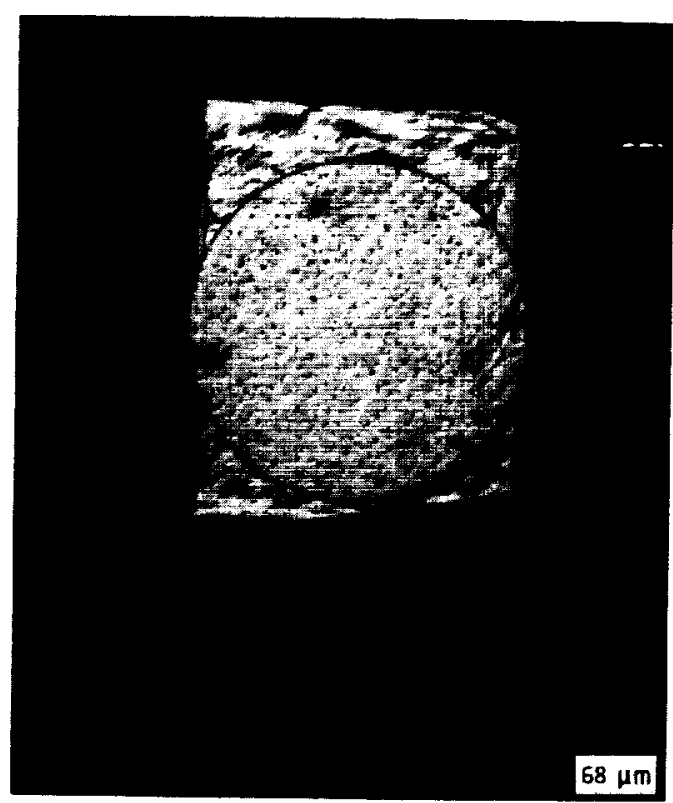

FIGURE 4. - ATTENUATION OF SPREADING FROM AN UNCAPPED DISC UPON ENCOUNTERING A CAP ON THE INP SURFACE. 


\section{ORISINAL PAGE \\ BLACK AND WHITE PHOIOGRAPH}

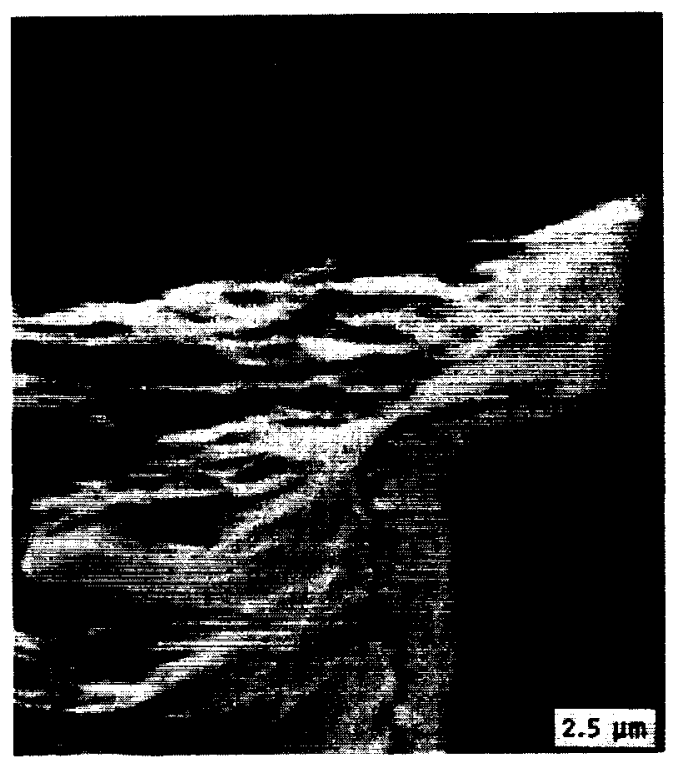

FIGURE 5. - TOPOGRAPHY OF SPREAD METALLIIZATION UNDER $\mathrm{SiO}_{2}$ CAP (CAP REMOVED).

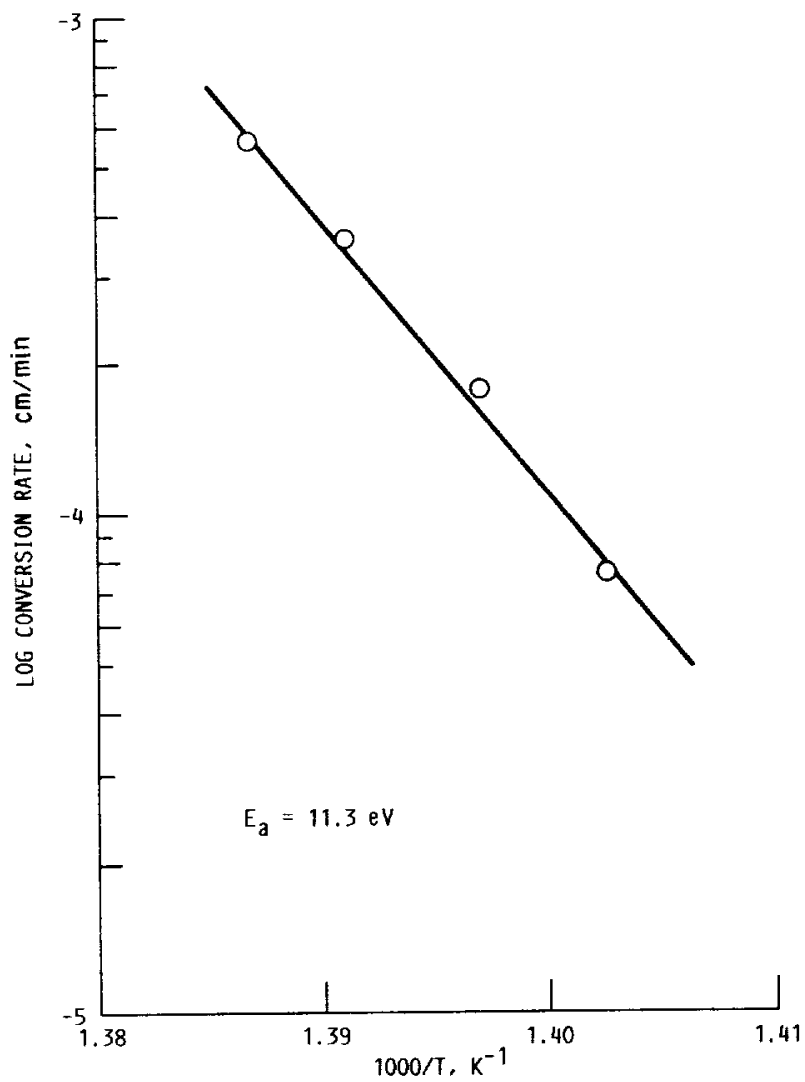

FIGURE 6. - EFFECT OF TEMPERATURE ON THE Au 3 In-T0-Aug $\mathrm{In}_{4}$ CONVERSION RATE. 


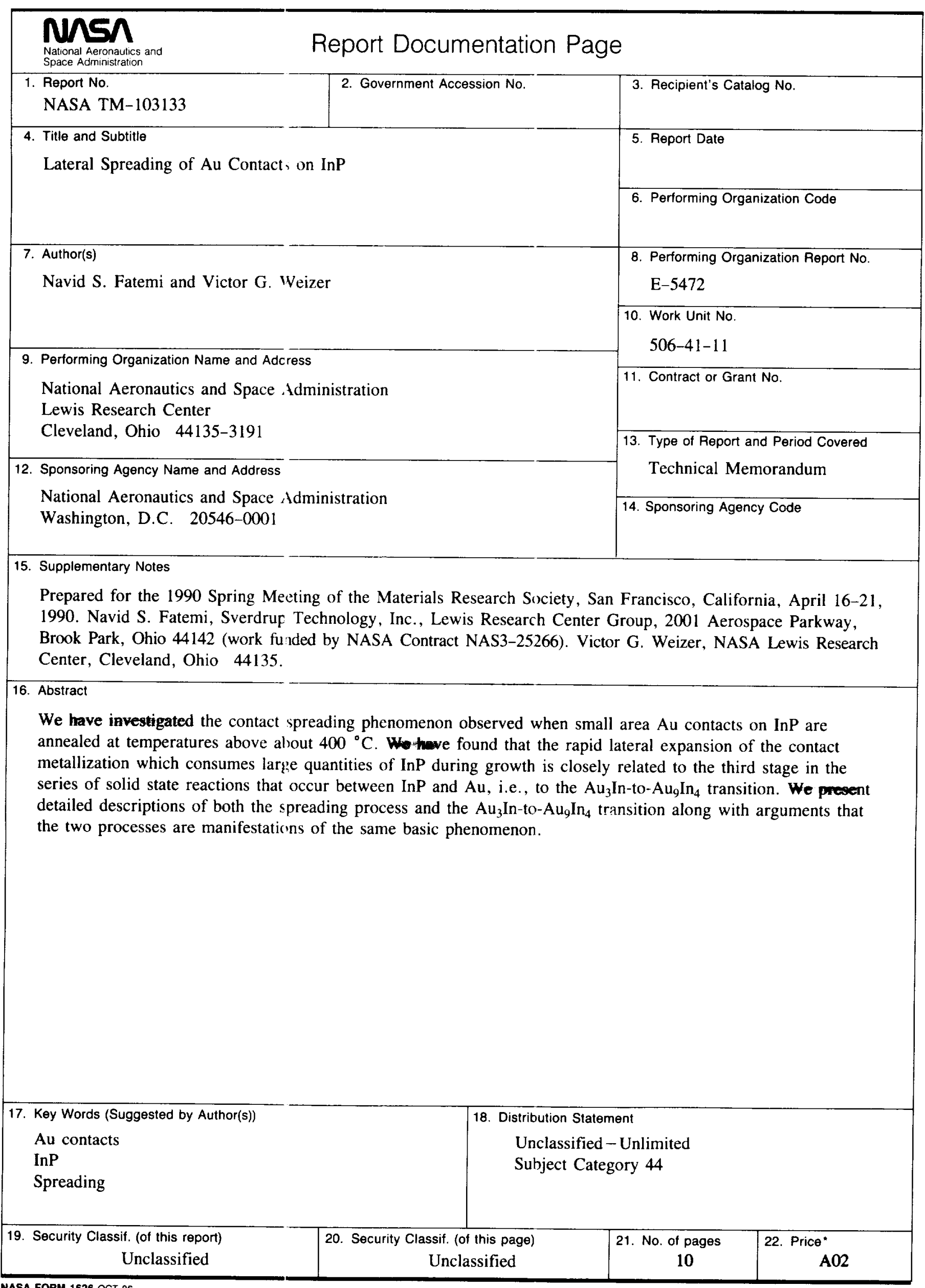


